\title{
EFICIÊNCIA DO USO E RESPOSTA À APLICAÇÃO DE FÓSFORO DE CULTIVARES DE ARROZ EM SOLOS DE TERRAS ALTAS ( $\left.{ }^{1}\right)$
}

\author{
ELIANE APARECIDA ROTILI $\left({ }^{2}\right)$; RODRIGO RIBEIRO FIDELIS $\left(3^{*}\right)$; MANOEL MOTA DOS SANTOS $\left({ }^{3}\right)$; \\ HÉLIO BANDEIRA BARROS (3); LEANDRO CARDOSO PINTO ( ${ }^{(3)}$
}

\begin{abstract}
RESUMO
Objetivou-se com este trabalho estudar a eficiência e resposta quanto ao uso de fósforo de cultivares de arroz em solos de terras altas no sul do Estado de Tocantins. Os tratamentos envolveram seis cultivares comerciais de arroz (BRS-Bonança BRS-Caiapó, BRS-Sertaneja, BRSMG-Curinga, BRSMG-Conai e BRS-Primavera), que foram cultivadas em dois ambientes distintos. Para simular ambientes com baixo e alto nível de fósforo, foram utilizadas as doses 20 e $120 \mathrm{~kg} \mathrm{ha}^{-1}$ de $\mathrm{P}_{2} \mathrm{O}_{5}$. O delineamento experimental utilizado foi o de blocos casualizados, com quatro repetições. Através da produtividade de grãos, classificaram-se as cultivares quanto à eficiência no uso e resposta à aplicação do fósforo. A cultivar BRS-Bonança foi mais eficiente quanto ao uso de fósforo e responsiva à sua aplicação.
\end{abstract}

Palavras-chave: Oryza sativa, genótipos, estresse mineral.

\author{
ABSTRACT \\ USE EFFICIENCY AND RESPONSE TO PHOSPHORUS OF APPLICATION \\ RICE CULTIVARS IN HIGHLANDS SOILS
}

This research's objective was to study the efficiency in the usage of phosphorus by rice cultivars in highland soils in the South of Tocantins State. The experiments evaluated six commercial cultivars of rice (BRS-Bonança BRSCaiapó, BRS-Sertaneja, BRSMG-Curinga, BRSMG-Conai and BRS-Primavera), grown in areas with high and low phosphorus application: 20 and $120 \mathrm{~kg} \mathrm{ha}^{-1}$ of $\mathrm{P}_{2} \mathrm{O}_{5}$. The experiments were carried out in a completely randomized blocks design, with four replicates. Based on grain yield, cultivars were classified in respect to the efficiency and response to phosphorus fertilization. The cultivar BRS-Bonança presented the highest phosphorus use efficiency and was the most responsive.

Key words: Oryza sativa, genotypes, mineral stress.

( $\left.{ }^{1}\right)$ Parte da Dissertação de Mestrado em Produção Vegetal do primeiro autor. Recebido para publicação em 4 de novembro de 2009 e aceito em 14 de maio de 2010.

(²) Mestre em Produção Vegetal, Universidade Federal do Tocantins (UFT), Campus Universitário de Gurupi, $77402-970$ Gurupi (TO). E-mail: elianerotili@yahoo.com.br

(3) Universidade Federal do Tocantins (UFT), 77.402-970 Gurupi (TO). E-mail: fidelisrr@uft.edu.br (*) Autor correspondente; barroshb@uft. edu.br; leandrouft@terra.com.br 


\section{INTRODUÇÃO}

O arroz (Oryza sativa L.) é considerado o produto de maior importância econômica em muitos países em desenvolvimento (constituindo-se alimento básico para cerca de 2,4 bilhões de pessoas) e o aumento crescente de seu consumo impõe aos setores produtivos busca de novas técnicas que possam aumentar a produção. Cultivado e consumido em todos continentes, o arroz se destaca pela produção e área de cultivo, desempenhando papel estratégico tanto em nível econômico quanto social (FAGERIA et al., 1997).

No Brasil, o arroz é produzido nos ecossistemas de várzea $(34 \%)$ e de terras altas $(60 \%)$ sob diversos sistemas de cultivo. O de terras altas apesar de ocupar $64 \%$ da área cultivada, responde por apenas 39\% da produção nacional, em razão da baixa produtividade média (StONE et al., 2001). No Tocantins, esta cultura faz-se presente tanto em várzea quanto em terras altas distribuído em todo o Estado. Na safra 2006/2007, a produção de arroz em terras altas foi de 168812 toneladas, em 100361 hectares, alcançando produtividade média de $1682 \mathrm{~kg} \mathrm{ha}^{-1}$ (SEAGro, 2009).

As limitações na disponibilidade de fósforo $(\mathrm{P})$ no início do ciclo vegetativo podem resultar em restrições no desenvolvimento, das quais a planta não se recupera posteriormente, mesmo aumentando o suprimento de $\mathrm{P}$ a níveis adequados. $\mathrm{O}$ suprimento adequado de $\mathrm{P}$ é, pois, essencial desde os estádios iniciais de crescimento da planta (TANGUilig et al., 1987).

A seleção de genótipos com maior eficiência na utilização de $\mathrm{P}$ é considerada uma das maneiras mais adequadas para diminuir o custo de produção da cultura do arroz (FAgeria e BARBosa FilHo, 1982; LuCA, 2002), posto que os genótipos de uma mesma espécie revelam exigências nutricionais e tolerâncias diferenciadas para os estresses de nutrientes essenciais, segundo (FAGERIA e BARBosa Filho, 1981).

Dessa forma, objetivou-se com este trabalho estudar a eficiência e resposta quanto ao uso de fósforo de cultivares de arroz em solos de terras altas no sul do Estado de Tocantins.

\section{MATERIAL E MÉTODOS}

Os experimentos (baixo e alto fósforo) foram realizados em terras altas, na fazenda Chaparral, no município de Gurupi, situada a $11^{\circ} 43^{\prime}$ de latitude sul e $49^{\circ} 15^{\prime}$ de longitude oeste, em solo do tipo Latossolo Vermelho-Amarelo Distrófico de classe textural arenosa, na safra 2007/2008.

O preparo do solo foi realizado da forma convencional, com duas gradagens sendo uma com grade "pesada" e outra com grade niveladora. A semeadura foi realizada em 13 de dezembro de 2007, manualmente, após abertura dos sulcos. A adubação de semeadura foi realizada no sulco de plantio com base nos resultados da análise química e física do solo. A análise química dos solos na camada de $0-20 \mathrm{~cm}$ de profundidade revelou os seguintes resultados: $\mathrm{pH}$ em $\mathrm{CaCl}_{2}=4,3 ; \mathrm{M} . \mathrm{O}(\%)=0,2 ; \mathrm{P}($ Melich $)=2,4 \mathrm{mg} \mathrm{dm}^{-3}$; $\mathrm{Ca}=0,9 \mathrm{cmol}_{c} \mathrm{dm}^{-3} ; \mathrm{Mg}=0,4 \mathrm{cmol}_{\mathrm{c}} \mathrm{dm}^{-3} ; \mathrm{H}+\mathrm{Al}=$ $2,9 \mathrm{cmol}_{\mathrm{c}} \mathrm{dm}^{-3}$ e $\mathrm{K}=0,06 \mathrm{cmol}_{\mathrm{c}} \mathrm{dm}^{-3}$.

O delineamento experimental utilizado foi o de blocos casualizados com quatro repetições. Cada unidade experimental foi constituída por cinco linhas de 5,0 $\mathrm{m}$ de comprimento, espaçadas de $0,45 \mathrm{~m}$ e semeando-se 60 sementes por metro linear. Como área útil foram utilizadas as três linhas centrais com 4,0 metros de comprimento, desprezando-se as duas linhas laterais e 0,5 metros das extremidades das três linhas centrais $\left(5,4 \mathrm{~m}^{2}\right.$ de área útil). Para o estudo, foram utilizadas seis cultivares de arroz recomendadas para o estado do Tocantins: BRS-Bonança, BRS-Caiapó, BRS-Sertaneja, BRSMG-Curinga, BRSMG-Conai e BRSPrimavera.

Para simular ambientes com baixo e alto nível de fósforo, foram utilizadas as doses de 20 e $120 \mathrm{~kg} \mathrm{ha}^{-1}$ de $\mathrm{P}_{2} \mathrm{O}_{5}$ no plantio, descontado o $\mathrm{P}$ do solo detectado na análise química, em ambientes distintos. Conforme FAGERIA (1991), estas duas doses contrastantes de fósforo foram identificadas em experimentos anteriores, para discriminar as cultivares de arroz quanto ao uso de P. O potássio foi aplicado em plantio na dosagem de $60 \mathrm{~kg}$ ha $^{-1}$ de $\mathrm{K}_{2} \mathrm{O}$ na forma de cloreto de potássio. A adubação de cobertura foi realizada em duas etapas, ambas com $45 \mathrm{~kg} \mathrm{ha}^{-1}$ de $\mathrm{N}$ aplicada por ocasião do perfilhamento efetivo e diferenciação do primórdio floral.

Os tratos fitossanitários foram efetuados quando necessários e o controle de plantas daninhas realizado mediante capina manual, sempre antes das adubações.

Para a análise estatística, utilizou-se o programa SISVAR (FERREIRA, 2000). A variável produtividade de grãos foi submetida à análise de variância com aplicação do teste $\mathrm{F}$ e para testar a significância dos tratamentos utilizou-se o teste de Tukey $(\mathrm{p}<0,05)$.

Para diferenciação das cultivares, utilizou-se a metodologia proposta por FAGERIA e KLUTHCOUSKI (1980) e FAGERIA e BALIGAr (1993), que sugerem a classificação das cultivares quanto à eficiência no uso e resposta à aplicação do fósforo (eficiência e resposta - ER). Na qual a utilização do nutriente é definida pela média de produtividade de grãos em baixo nível. A resposta à utilização do nutriente é obtida pela diferença entre a produtividade de grãos nos dois níveis dividida pela diferença entre as doses utilizando a seguinte fórmula: 


$$
\alpha=(\mathrm{PNN}-\mathrm{PBN}) / \mathrm{DEN},
$$

em que:

PNN = Produção com nível ideal de nutriente;

PBN = Produção com baixo nível do nutriente, e;

$\mathrm{DEN}=$ Diferença entre as doses $\left(\mathrm{kg} \mathrm{ha}^{-1}\right)$.

Foi utilizada uma representação gráfica no plano cartesiano para classificar as cultivares. No eixo das abscissas (y), está a eficiência na utilização do fósforo e no eixo das ordenadas (x), a resposta à sua utilização. O ponto de origem dos eixos é a eficiência média e a resposta média das cultivares. No primeiro quadrante, são representadas as cultivares eficientes e responsivas (ER); no segundo, as não-eficientes e responsivas (NER); no terceiro, as não-eficientes e não-responsivas (NENR) e no quarto, as eficientes e não-responsivas (ENR).

\section{RESULTADOS E DISCUSSÃO}

$\mathrm{O}$ presente trabalho identificou como eficiente no uso de fósforo as cultivares BRS-Bonança, BRSSertaneja e BRS-Caiapó, pois nestas houve as maiores médias de produtividades de grãos quando cultivadas em ambientes de baixo P, portanto, estão representadas no primeiro e quarto quadrante da figura 1 . A eficiência dessas cultivares, em relação às demais, na absorção e utilização de $\mathrm{P}$ na produção de grãos, permite inferir que os processos associados à absorção, translocação, assimilação e redistribuição de $\mathrm{P}$ são mais eficientes do que nas demais cultivares. Segundo Matias (2006), plantas eficientes na absorção de $\mathrm{P}$, são aquelas que acumulam maiores quantidades do elemento quando cultivadas em baixo nível de P.

Quanto à resposta a aplicação de $\mathrm{P}$, os maiores índices foram obtidos pelas cultivares BRS-Bonança, BRS-Primavera e BRSMG-Conai e estão representadas no primeiro e segundo quadrante da figura 1. Destes, somente a cultivar BRS-Bonança proporcionou valor de índice de resposta acima de 2 ou seja, acresce dois quilogramas de grãos para cada quilograma de $\mathrm{P}$ aplicado (Tabela 1).

Desta forma, apenas a cultivar BRS-Bonança (moderna) foi considerada eficiente quanto ao uso e responsiva à aplicação de fósforo, sendo, portanto, recomendada para cultivo em propriedades que adotam desde baixo, médio a alto nível tecnológico (insumos). Além de produzir razoavelmente bem em ambientes desfavoráveis (baixo $\mathrm{P}$ ), é responsiva à melhoria do ambiente, com o incremento de adubação (alto P), resultando em aumento na produção equivalente a $271 \mathrm{~kg}$ ha $^{-1}$ (Tabela 1). Esta cultivar proporcionou produtividade média tanto em ambiente de baixo quanto em ambiente de alto $\mathrm{P}$ acima da média de produtividade do Estado do Tocantins na safra 2006/2007, que foi de $1682 \mathrm{~kg} \mathrm{ha}^{-1}$ (Seagro, 2009).

Estes dados discordam, porém, daqueles observados por MATIAS (2006), que avaliou em casa de vegetação a eficiência de absorção e utilização de $\mathrm{P}$ em cultivares de arroz de terras altas, tradicionais e modernas e classificou a cultivar BRS-Bonança como não-eficiente quanto ao uso e responsiva à aplicação de fósforo.

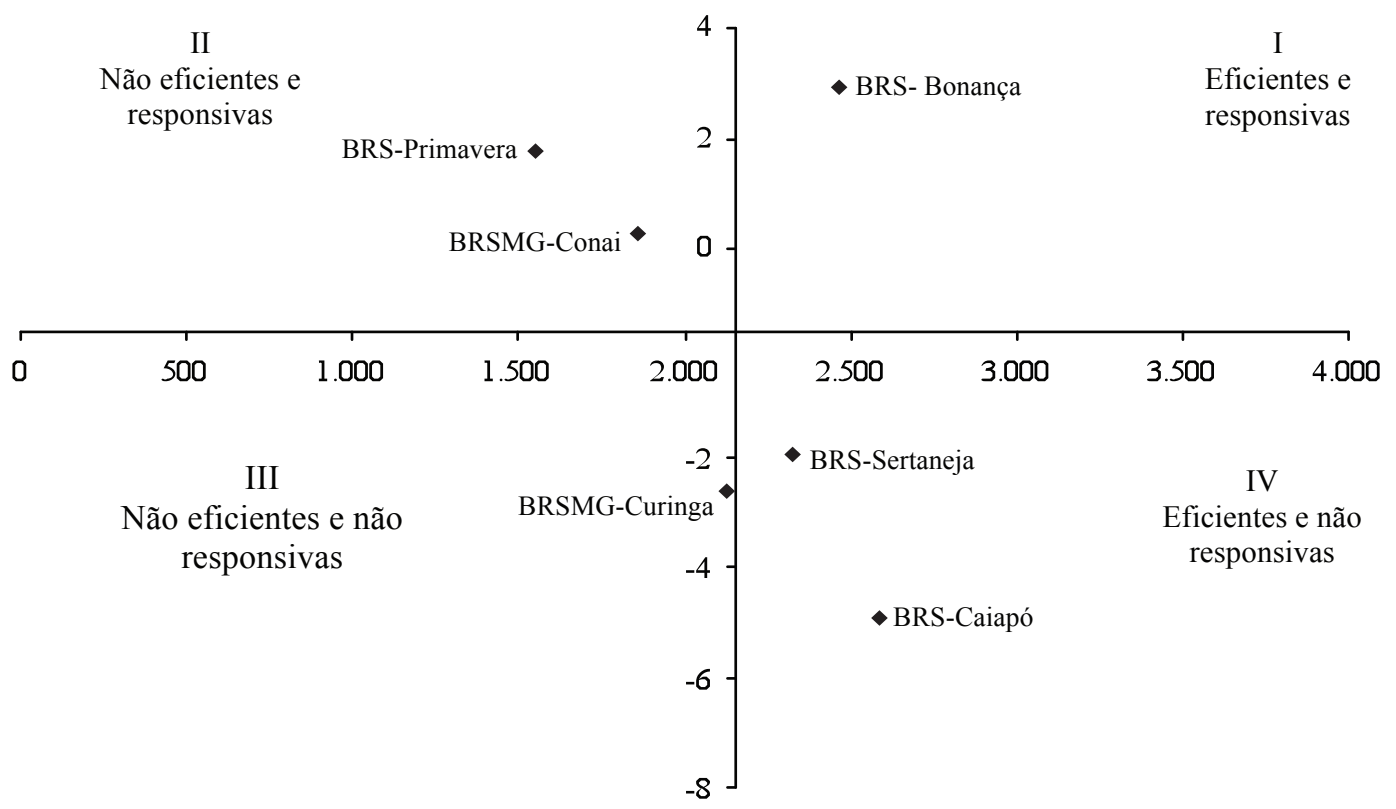

Figura 1. Eficiência no uso e resposta à aplicação de fósforo em cultivares de arroz, pela metodologia de FAGERIA e KLUTHCOUSKI (1980). 
Tabela 1. Média da característica produtividade de grãos, com baixa e alta dose de P (20 e $120 \mathrm{~kg} \mathrm{ha}^{-1}$ de $\mathrm{P}_{2} \mathrm{O}_{5^{\prime}}$, respectivamente) para cultivares de arroz, cultivado em terras altas na região sul do Estado de Tocantins, Gurupi, (TO), safra 2007/2008

\begin{tabular}{|c|c|c|c|c|c|}
\hline Cultivares & Baixo P & Alto P & Média & Diferença de produção & Índice de Resposta \\
\hline & & & $\mathrm{kg} \mathrm{ha}^{-1}$ & & \\
\hline BRS-Bonança & 2458 & 2729 & 2594 & 271 & 2,71 \\
\hline BRS-Caiapó & 2590 & 1912 & 2251 & 678 & $-6,78$ \\
\hline BRS-Sertaneja & 2330 & 1951 & 2140 & -379 & $-3,79$ \\
\hline BRSMG-Curinga & 2127 & 1826 & 1976 & -301 & $-3,01$ \\
\hline BRSMG-Conai & 1847 & 1875 & 1861 & 28 & 0,28 \\
\hline BRS-Primavera & 1552 & 1717 & 1635 & 165 & 1,65 \\
\hline Média & 2151 & 2002 & 2076 & -149 & $-1,49$ \\
\hline C.V. (\%) & & & 24,53 & & \\
\hline
\end{tabular}

As cultivares BRS-Primavera e BRSMG-Conai foram consideradas como não eficientes e responsivas, pois produziram abaixo da média dos genótipos sob ambiente de baixo nível de fósforo, porém tiveram valores de índice de resposta acima da média geral de índice de resposta $(-1,49)$, resultando em aumento da produtividade equivalente a 165 e $28 \mathrm{~kg} \mathrm{ha}^{-1}$ respectivamente (Tabela 1). Cultivares do grupo não eficientes e responsivas são as mais indicadas aos produtores que dispõem de elevado nível tecnológico. Esses resultados corroboram com os de Matias (2006), que também classificou a cultivar BRS-Primavera como não-eficientes e responsivas.

A cultivar BRSMG-Curinga foi classificada como não-eficiente e não- responsiva, pois produziu abaixo da média em ambiente de baixo e alto nível de P. De acordo com a metodologia utilizada, cultivares classificadas como não-eficiente e não- responsiva não são recomendadas para serem semeadas em propriedades agrícolas, nem mesmo para aquelas que utilizam baixo nível tecnológico. Esses resultados discordam dos constados por Matias (2006), que classificou esta cultivar em seu estudo como eficientes e responsivas. Resultados verificados em casa de vegetação, como os de Matias (2006), não devem ser extrapolados para o campo uma vez que em vasos o sistema radicular não tem o mesmo desenvolvimento que no campo e a absorção de Pi depende do volume de solo explorado (FAGERIA et al., 1997).

No quadrante das eficientes e não responsivas estão as cultivares BRS-Sertaneja e BRS-Caiapó, que produziram acima da média em ambiente com baixo $\mathrm{Pe}$ abaixo da média em ambientes de alto P. O que evidencia a falta de resposta à melhoria do ambiente com o incremento do nutriente. Cultivares do grupo eficientes e não responsivas são recomendadas para o cultivo em propriedades que adotam baixo nível tecnológico. Esses resultados assemelham-se aos de Matias (2006), que também classificou a cultivar BRS-Caiapó, em seu estudo como eficientes e não responsivas.
Das cultivares avaliadas, a BRS-Caiapó é a única considerada tradicional ou não melhorada, pertencendo as demais ao grupo de cultivares melhoradas ou modernas. Esse fato pode ser evidenciado analisando a tabela 1, mais especificamente o ambiente de baixo P. Verifica-se que a cultivar BRS-Caiapó obteve maior valor de produtividade de grãos quando comparada com os demais genótipos, evidenciando melhor adaptação devido à provável rusticidade. Resultados semelhantes foram também constatados por CRUSCIOL et al. (2005), que observaram nas cultivares Carajás e IAC 201, classificadas como tradicionais, maior habilidade em relação às cultivares do grupo moderno em se desenvolverem na ausência de adubação fosfatada. De acordo com TiffNEY e NiKLAS (1985), esse comportamento está associado à maior adaptação às condições adversas que as cultivares do grupo tradicional em relação ao grupo moderno; assim como, a capacidade do sistema radicular em alterar sua configuração geométrica, de forma que melhor explore o solo em busca de nutrientes ou de adquirir os recursos necessários de maneira mais eficiente. SANT' ANA et al. (2003) relataram que cultivares tradicionais são materiais mais adaptados às condições adversas dos solos de cerrados e têm mostrado serem eficientes na extração do $\mathrm{P}$ em solos pobres desse nutriente.

As cultivares modernas avaliadas nesse estudo proporcionaram baixas médias de produtividade, pois não foram responsivas ao acréscimo de $\mathrm{P}$, discordando de FAGERIA et al. (1995), que afirmam que cultivares do grupo moderno necessitam de níveis mais elevados de fósforo para atingirem a máxima produtividade. Quando ocorre o fornecimento de fósforo há maior acúmulo de matéria seca pela planta, evidenciando maior exigência e respostas à melhoria da fertilidade do solo em relação às cultivares do grupo intermediário e tradicional.

As médias de produtividade de grãos das cultivares em ambientes de baixo e alto P foram de $2151 \mathrm{~kg} \mathrm{ha}^{-1}$ e $2002 \mathrm{~kg} \mathrm{ha}^{-1}$ respectivamente, superiores às obtidas por GUIMARÃES et al. (2007) que avaliando em 
campo 51 linhagens de arroz em condições de baixo e alto $\mathrm{P}$, obtiveram produtividade média de $1619 \mathrm{~kg} \mathrm{ha}^{-1}$ no tratamento com alto e $478 \mathrm{~kg} \mathrm{ha}^{-1}$ no tratamento de baixo P. CRUSCIOL et al. (2003) relataram em seu estudo que as diferenças observadas na literatura quanto à absorção e utilização de nutrientes estão relacionadas às diferenças existentes entre as cultivares utilizadas.

\section{CONCLUSÃO}

A cultivar BRS-Bonança foi eficiente quanto ao uso de fósforo e responsiva à sua aplicação.

\section{AGRADECIMENTOS}

À Universidade Federal do Tocantins - UFT, ao CT-INFRA por intermédio do $\mathrm{MCT} / \mathrm{CNPq}$ pela concessão de bolsa de iniciação científica. À Secretaria de Ciência e Tecnologia - CECT, pelo apoio financeiro para execução do projeto. À Fazenda Chaparral, pela doação da área para realização dos experimentos.

\section{REFERÊNCIAS}

CRUSCIOL, C.A.C.; ARF, O.; SORATTO, R.P.; ANDREOTTI, M.; RODRIGUES, R.A.F. Absorção, exportação e eficiência de utilização de nutrientes pela cultura do arroz de terras altas em função de lâmina de água aplicadas por aspersão. Acta Scientiarum Agronomy, v.25, p.97-102, 2003.

CRUSCIOL, C.A.C.; MAUAD, M.; CASSIA, R.; ALVAREZ, F.; LIMA, E.V.; TIRITAN, C.S. Doses de fósforo e crescimento radicular de cultivares de arroz de terras altas. Bragantia, v.64, p.643-649, 2005.

FAGERIA, N.K. Resposta de cultivares de arroz a fertilizante fosfatado em Latossolo Vermelho Escuro do Brasil Central. Revista Brasileira de Ciência do Solo, v.15, p.63-67, 1991.

FAGERIA, N.D.; KLUTHCOUSKI, J. Metodologia para avaliação de cultivares de arroz e feijão para condições adversas de solo. Brasília: EMBRAPA/CNPAF, 1980. 22p.

FAGERIA, N.K.; BALIGAR, V.C. Screening crop genotypes for mineral stresses. In: WORKSHOP ON ADAPTATION OF PLANTS TO SOIL STRESSES, 1993, Lincoln. Proceedings... Lincoln: University of Nebraska, 1993. p.142-159. (Intsormil Publication, 94-2).

FAGERIA, N.K.; BALIGAR, V.C.; JONES, C.A. Growth and mineral nutrition of field crops. 2.ed. New York: Marcel Dekker, 1997. 624p.
FAGERIA, N.K.; BARBOSA FILHO, M.P. Avaliação de cultivares de arroz em função de sua tolerância ao baixo nível de fósforo disponível do solo. Revista Brasileira de Ciência do Solo, v.6, p.146-151, 1982.

FAGERIA, N.K.; BARBOSA FILHO, M.P. Avaliação de cultivares de arroz para maior eficiência na absorção de fósforo. Pesquisa Agropecuária Brasileira, v.16, p.777-782, 1981.

FAGERIA, N.K.; SANT'ANA, E.P.; MORAES, O.P. Resposta de genótipos de arroz de sequeiro favorecido à fertilidade do solo. Pesquisa Agropecuária Brasileira, v.30, p.1155-1161, 1995.

FERREIRA, D.F. Sistema de análises de variância para dados balanceados. Lavras: UFLA, 2000. (SISVAR 4.1. pacote computacional).

GUIMARÃES, C.M.; STONE, L.F.; NEVES, P.C.F. Resposta de arroz de terras altas ao estresse de fósforo no solo. Revista Brasileira de Engenharia Agrícola e Ambiental, v.11, p.578584, 2007.

LUCA, E.F.; BOARETTO, A.E.; MURAOKA, T.; CHITOLINA, J.C. Eficiência de absorção de fósforo (P) por mudas de eucalipto e arroz. Scientia Agricola, v.59, p.543-547, 2002.

MATIAS, G.C.S. Eficiência nutricional de fontes de fósforo com solubilidade variável em água em cultivares de arroz (Oryza sativa L.). 2006. 93f. Dissertação (Mestrado em Solos e Nutrição de Plantas) - Universidade de São Paulo, São Paulo.

SANT'ANA, E.P.; SANT'ANA, E.V.P.; FAGERIA, N.K.; FREIRE, A.B. Utilização de fósforo e características do sistema radicular e da parte aérea da planta de arroz. Ciência e Agrotecnologia, v.27, p.370-381, 2003.

SECRETARIA DA AGRICULTURA, PECUÁRIA E ABASTECIMENTODOESTADODO TOCANTINS (SEAGRO). Evolução da Produção do Arroz. Disponível em: <http:/ / central2.to.gov.br/arquivo/14/100>. Acesso em 30 abr. 2009.

STONE, L.F.; MOREIRA, J.A.A.; RABELO, R.R.; BIAVA, M. Arroz: O produtor pergunta, a Embrapa responde. Brasília: Embrapa Arroz e Feijão: Embrapa informação tecnológica, 2001. 232p.

TANGUILIG, V.C.; YAMBAO, E.B.; O' TOOLE, J.C.; DATTA, S.K. Water stress effects on leaf elongation, leaf water potential, transpiration, and nutrient uptake of rice, maize, and soybean. Plant and Soil, v.103, p.155-168, 1987.

TIFFNEY, B.H.; NIKLAS, K.J. Clonal growth in land plants: a palaeobotanical prespective. In: JACKSON, J.B.C.; BUSS, L.W.; COOK, R.E (Ed). Population biology and evolution of clonal organisms. New Haven: Yale Universiry Press, 1985. p.35-66. 